DOI: $10.4274 /$ jarem.galenos.2021.3980

J Acad Res Med 2021;11(2):149-56

\title{
A New Algorithm for Dynamic Vestibular System Analysis with Wearable Pressure and Motion Sensors
}

\author{
(1) Eyyup Kara1, (1) Serhat İkizoğlu22, (1) Kaan Şahin2, (1) Tunay Çakar2, (1) Ahmet Ataş3 \\ ${ }^{1}$ istanbul University-Cerrahpaşa, Faculty of Health Science, Department of Audiology, İstanbul, Turkey

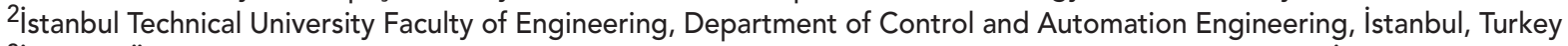 \\ ${ }^{3}$ İstanbul Üniversitesi-Cerrahpaşa, Cerrahpaşa Faculty of Medicine, Department of Otorhinolaryngology, İstanbul, Turkey
}

Cite this article as: Kara E, İkizoğlu S, Şahin K, Çakar T, Ataş A. A New Algorithm for Dynamic Vestibular System Analysis with Wearable Pressure and Motion Sensors. J Acad Res Med 2021;11(2):149-56

\begin{abstract}
Objective: Balance is a complex state that requires integrating information from many systems into various levels of the nervous system and the actions of commands from the central nervous system and the musculoskeletal system. Postural control is the ability to maintain body position against forces that threaten the individual's orientation or balance. In our study, a mobile prototype system was used to create equilibrium data of subjects and generate a set of normal values, diseases and fuzzy decisions.

Methods: This study included 106 adults (55 females, 51 males) with no vestibular, neurologic, orthopaedic and eye problems to create the normalisation data. Also, 60 patients (37 females, 23 males) with different vestibular pathologies (23 benign paroxysmal positional vertigo, 12 Meniere's disease, 10 vestibular neuritis, 15 unilateral vestibular hypofunction) comprised the patient group.

Results: With the newly developed system, step length and symmetry, distance between feet, gait symmetry, ankle mobility and symmetry and phases of each step and step symmetry were collected. Significant differences were found between the normal and pathological groups in the data obtained from the dynamic balance and pressure sensors on the sole of the foot.

Conclusion: Dynamic balance and walking analysis by our system are considered important in audiology, orthopaedics and neurology. It can contribute clinically by collecting data from several patients with different pathologies.
\end{abstract}

Keywords: Balance, posture, mobile vestibular algorithm, motion and pressure sensors, motion/walking analysis

\section{INTRODUCTION}

Balance is a complex state that requires the integration of information from many systems into various levels of the nervous system and the actions of commands from the central nervous system and the musculoskeletal system (1). The regulation of sensory stimuli to provide vertical posture is complex and related to perception and movement planning (2). While vision, perception and the vestibular system (VS) provide detailed information about the environment, the coordinated motor response occurs through spinal stretch reflexes and long latency reflexes (3). Body posture and position "postural adjustments", which are static or dynamic, maintain balance (4).

Postural control is the ability to maintain body position against forces that threaten the individual's orientation or balance (5). The vestibular, visual and somatosensory systems are effective in normal postural control and stabilisation (6). All these systems are under the influence of varying environmental conditions of

ORCID IDs of the authors: E.K. 0000-0002-4015-4560; S.I. 0000-0003-2394-7988; K.Ş. 0000-0003-3886-6187; T.Ç. 0000-0001-6440-5814; A.A. 00000002-8673-6793. 
the neck, lower extremity and trunk muscles allow the body to maintain an upright position (7).

Current systems to evaluate balance usually use a fixed platform in the clinical setting and are based on data evaluated from plantar measures. Video nystagmography (VNG) and dynamic posturography are used in the clinical setting but are costly for clinics. The mobile systems used in recent years are aimed only to monitor body movements.

In today's world, systems are getting smarter every day. The system first learns about a particular issue and then decides how to react depending on the case and/or classifies the new data. This whole procedure is called machine learning $(\mathrm{ML})$. The key point here is to develop the proper algorithm to teach the machine how to attain high overall accuracy. In this study, we took substantial steps to develop an algorithm to identify the reason behind the balance disorder of the patient and differentiated between VS dysfunction-based diseases. Finally, we aim to develop a dynamic VS analysis algorithm and produce a balance detector (BD) to determine the balance performance of the patient with high accuracy in the diagnosis and treatment of vestibular disorders by measuring postural control and static/dynamic balance parameters via wearable sensors.

The purpose of our study is to measure postural control, static and dynamic balance parameters with portable equipment on the patient. It aims to develop a dynamic VS analysis algorithm and produce a BD to determine the balance performance of the patient with high accuracy in the diagnosis and treatment of vestibular disorders. In clinical practice, especially in pathologies with difficult decision-making challenges, a ML model can make the correct diagnosis using the findings of gait and body swing limits to create an algorithm and hardware that efficiently process the different data obtained and make small changes that clinicians have difficulty seeing.

\section{METHODS}

This study included 106 adults ( 55 females, 51 males; ages ranging between 25 and 60 years; mean, 45.1 years) with no vestibular, neurologic, orthopaedic and eye problems from normalisation data and 60 patients (37 females, 23 males; ages ranging between 27 and 60, mean 47.3 years) with different vestibular pathologies [23 benign paroxysmal positional vertigo (BPPV), 12 Meniere's disease (MD), 10 vestibular neuritis (VN), 15 unilateral vestibular hypofunction (UVH)] comprised the patient group at İstanbul University-Cerrahpaşa, Cerrahpaşa Medical Faculty, ENT/ Audiology Unit. The study has been approved by the institutional review board with the approval number A-57/07.07.2015, and written consent was obtained from the participants before the study.

Middle ear systems and acoustic reflexes were tested with GSI Tympstar (Grason-Stadler, USA) immittance meter while hearing tests were performed with GN Otometrcis Aurical (GN Otometrics,
Denmark) audiometry. Vestibulo-ocular and positional testing of the subjects were performed with GN Otometrics ICS Chartr 200 VNG (GN Otometrics, Denmark); vestibulo-spinal, dynamic balance assessments were carried out using Neurocom SMART Balance Master ${ }^{\circledR}$ (Natus Medical, USA) computerised dynamic posturography.

In general terms, the study involved the steps of 'data collection, data transmission, data processing and evaluation on the computer'. Technical equipment of the device was developed in İstanbul Technical University, Control \& Automation Department. İstanbul University-Cerrahpaşa, Audiology Department facilities were utilised to create a data bank, determine the correlation between data from various sensors and evaluate these medically and test the device on patients.

With the equipment developed; force sensors embedded in the insole (heel, toe, metatarsal area) and motion sensors on the body; data was collected to characterise the following discriminative features: Walking speed, average step length, step speed, step symmetry in the distance walked, toes motion characteristics during feet's lifting from the floor, the distance between steps, anterior-posterior swing angle of the body and maximum limits for the movement, anterior swing of the body, the posterior swing of the body, body tilt angle during gait, lateral swing limits of the body, the maximum bending angle of each knee during gait, lateral swing limits of each knee. Also, from the pressure sensors on the sole usage percentage of right heel, right medial forefoot, right lateral forefoot, right toe, left heel, right medial forefoot, left lateral forefoot, left toe during steps were calculated.

\section{Collecting Data Through a Newly Developed System}

A mobile prototype system was used to create equilibrium data of subjects and a set of normal values, disease and fuzzy decisions. The hardware block diagram of the system is shown in Figure 1. Special insole with piezoresistive analogue pressure sensors embedded appropriately (4 sensors/insole) to be worn under the foot was designed to obtain plantar pressure distribution during walking (Figure 2). Signals from plantar sensors were transferred to a data acquisition unit (Figure 1-DAQ1) located on the subject's body. Signals were amplified and conditioned, then converted into digital data via an analogue/digital converter. Data concerning body movements and the changing distance between legs when walking was obtained with motion and position sensors (Five XSens MTI-G (Xsens North America Inc. USA) (Figure 3) placed on the body. Data collected from the pressure and motion sensors were transmitted simultaneously to a computer via a second data acquisition unit using wireless communication (Figure 1-DAQ2).

In our study, in distinguishing two classes (in our case: The classes 'Healthy \& diseased', or two different diseases), correlation statistics can directly be used to speak of one-dimensional vectors. In our case, this corresponds to a single feature only. Thus, as an example, if we search for the separation ability of the 
feature 'stride length' between two diseases, we can apply the $\mathrm{p}$ value test. Nevertheless, in our study, we use several features; our vector is not one-dimensional but multi-dimensional.

In our work, the starting number of features was around 150. All these features were constituted by the data collected from eight pressure sensors (four under each foot) and five motion sensors (placed on the body). Examples for these features are: walking speed, average step length, use of forefoot (frequency and time-domain analysis of pressure data), correlations of all these features, others. Thus, on a mathematical basis, we cannot speak of a single point as the intersection of different classes but a spatial clustering in an $n$-dimensional space ( $n$ : number of features). We may not expect that every feature can distinguish between two classes (two diseases), but the whole set of features should perform the correct discrimination. This is exactly the point where $\mathrm{ML}$ helps.

We implemented a two-step classification algorithm. At the first step, the new subject was decided as belonging either to the healthy group or to the suffering. For this part of the procedure, we entered all data collected from normal and diseased subjects $(106+60=166$ subjects in total) to train the machine. The 5 -fold cross-validation $\mathrm{ML}$ resulted in the best accuracy of $99.4 \%$,

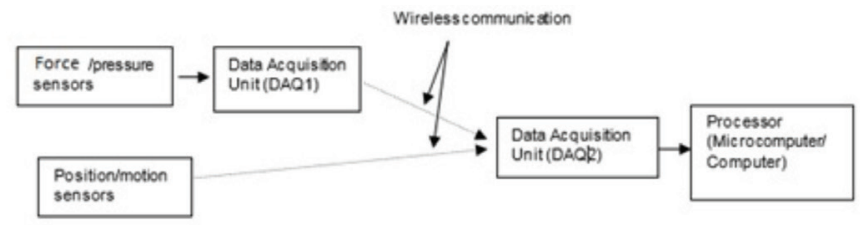

Figure 1. Block diagram of the mobile dynamic system
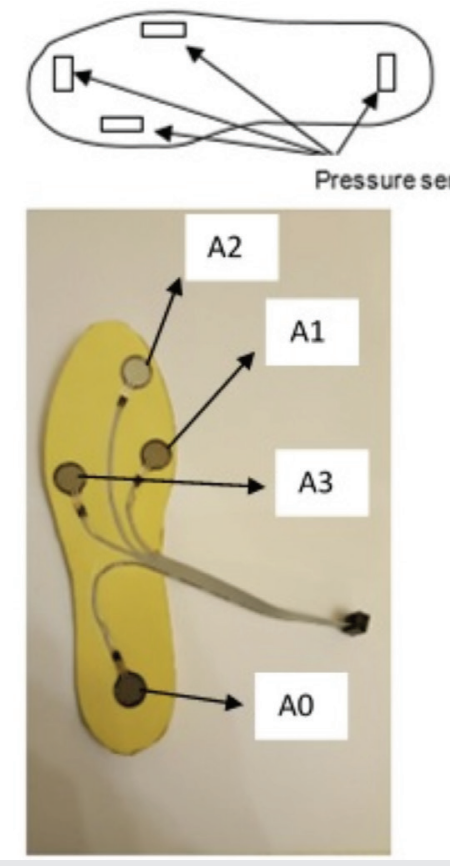

Figure 2. Positioning of insole pressure sensors and placement inside the insole achieved by applying the Support Vector Machines (SVM) method. This means that we reached $99.4 \%$ of success in distinguishing between healthy and vestibular disorders groups for new subjects.

As the second step of the classification, we tried to classify between different diseases in case the first step ended up with the decision 'Belonging to vestibular disorders group'. This time we trained the machine with data collected from the diseased subjects (60 subjects) only.

Combining the two steps resulted in the overall accuracy in determining the specific disease related to vestibular disorders was $99.4 \% * 94.4 \%=93.8 \%$.

Calculations for both steps were performed in a Matlab environment using classification tools. For that, we used another two-step algorithm. We first selected the most significant features by applying the 'dimensionality reduction' step, where the principal component analysis method performed best. As the second step, the machine was trained using these selected features.

\section{Collecting Vestibular/Walking Data}

The motion of the lower body was analysed during walking in the proposed system. This system included five sensor units in total. One unit was placed on each of the feet and knees. One was positioned on the back of the waist (Figure 3). An inertial measurement unit with an accelerometer, gyroscope and magnetometer (MTw Development Kit of Xsens) was used (8).

$M L$ was implemented in two stages: In the first stage, the values of normal subjects were obtained, loaded on the machine and learning of normality values was provided. Then, the data of individuals with pathology were obtained, loaded on the machine and the distinction between normal and pathological individuals was made. After this phase, by recognising the diseases within themselves, the classification was performed.

\section{First Stage}

Data from subjects were applied to the ML method. For this purpose, the data of 106 (normal subjects) and 60 patient subjects clusters were evaluated as education. The accuracy of the training models was determined with 5-fold "cross-validation" to prevent over-fitting.
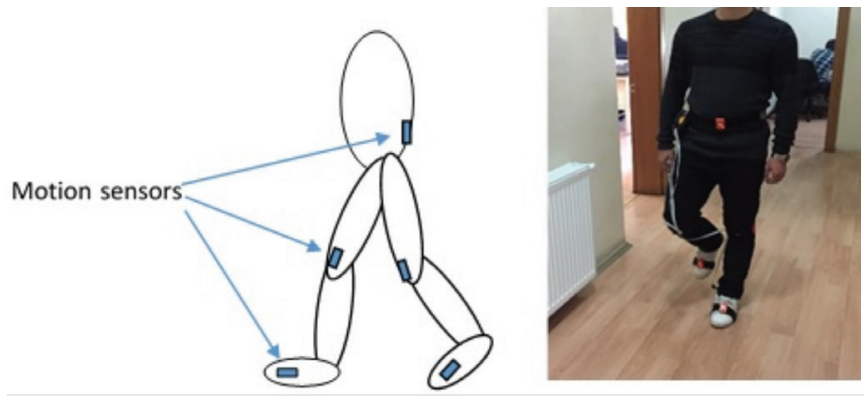

Figure 3. Several locations where motion/position sensors are placed on the body 


\section{Second Stage (Determination of Disease)}

Different algorithms were tried for this phase. As a patient set, data for 60 subjects belonging to BPPV, MD, VN and UVH sets were used. Measurements provided data to prefer SVM for ML. A two-step decision mechanism was operated for the diagnosis. In this framework, a double classification procedure was applied in the first stage only as patient-healthy. If the person was determined to be in the "patient" group, the disease was tried to be determined by moving to the second stage.

Gait parameters were processed with separate and integrated data, extracting the features that could affect balance. The data of normal subjects were compared with the data of the patient group, and the most significant values are presented. An analysis according to gender, weight and height was performed. Also, the side of the vestibular disorder according to different characteristics was calculated.

\section{Statistical Analysis}

The statistical analysis was performed using the SPSS 21 programme. The comparison of normal and patient groups was made by the Student's t-test, and correlations between normal and patients having BPPV, MD, VN and UVH were made by the Spearman correlation test.

\section{RESULTS}

The data relating to gait characteristics recorded from the body sensors of normal subjects and patients with various disorders are shown in Table 1, which demonstrates significant differences in these parameters. In Table 2, the data collected from sole pressure sensors from normal and patient subjects are presented. The comparison of average step length, step symmetry in the distance walked, step speed, back of the waist anterior-posterior, tilt angle during gait, back of the waist lateral swing maximum (max), max. left knee bending angle, max. left lateral swing, max. right knee bending angle, max. right lateral swing in normal subjects and patients with vestibular pathology were significantly different (Table 3).

Table 4 presents the correlation analysis of different gait characteristics recorded from body sensors. In this analysis, walking speed, average step length, step symmetry in the distance walked, step speed, back of the waist anterior swing, tilt angle during gait, max. left knee bending angle, max. left lateral swing, max. right knee bending angle, max. right lateral swing values of normal subjects and patients with BPPV were significantly correlated. Analysis of normal subjects and MD revealed a significant correlation with walking speed, average step length, step symmetry in the distance walked, average step width, back of the waist anterior-posterior, back of the waist anterior swing, back of the waist lateral swing right, back of the waist lateral swing max., max. left knee bending angle, max. left lateral swing, max. Right knee bending angle. The correlations between normal subjects and patients with VN revealed a significant difference in walking speed, average step length, step symmetry in the distance walked, step speed, average step width, back of the waist anterior-posterior, max. left knee bending angle, max. right knee bending angle. In UVH and normal subject analysis,

\section{Table 1. Averages gait parameters in normal subjects and patients with different vestibular pathologies}

\begin{tabular}{|c|c|c|c|c|c|}
\hline Gait Parameters & Normal & BPPV & Meniere's disease & Vestibular neuritis & $\begin{array}{l}\text { Unilateral vestibular } \\
\text { hypofunction }\end{array}$ \\
\hline Average step length (m) & 0.63 & 0.51 & 0.57 & 0.58 & 0.60 \\
\hline Step symmetry in the distance walked & 1.02 & 1.61 & 2.69 & 2.19 & 2.85 \\
\hline Step speed $(\mathrm{m} / \mathrm{s})$ & 1.36 & 1.03 & 1.13 & 1.13 & 1.18 \\
\hline Lifting height of the right foot $(\mathrm{m})$ & 0.11 & 0.09 & 0.11 & 0.09 & 0.10 \\
\hline Average step width (m) & 0.19 & 0.11 & 0.26 & 0.16 & 0.14 \\
\hline Back of the waist anterior-posterior (Deg) & 14.6 & 10.58 & 10.47 & 9.62 & 10.60 \\
\hline Back of the waist anterior swing (Deg) & 4.6 & 5.09 & 5.25 & 4.71 & 5.63 \\
\hline Back of the waist posterior swing (Deg) & 3.63 & 5.35 & 5.22 & 4.91 & 4.98 \\
\hline Back of the waist lateral swing max. (Deg) & 10.85 & 8.66 & 11.40 & 11.68 & 9.96 \\
\hline Max. left knee bending angle (Deg) & 65.64 & 55.84 & 43.34 & 45.65 & 50.89 \\
\hline Max. left lateral swing (Deg) & 13.44 & 19.21 & 15.68 & 16.69 & 16.62 \\
\hline Max. right knee bending angle (Deg) & 62.9 & 54.64 & 43.36 & 44.47 & 51.21 \\
\hline Max right lateral swing (Deg) & 12.9 & 20.18 & 16.95 & 18.52 & 17.48 \\
\hline
\end{tabular}




\begin{tabular}{|l|l|l|l|l|l|l|}
\hline \multicolumn{3}{|l}{ Table 2. Average plantar pressure parameters of normal subjects and patients with different vestibular pathologies } \\
\hline Plantar pressure parameters & Normal & BPPV & Meniere's disease & Vestibular neuritis & Unilateral vestibular hypofunction \\
\hline Right heel (A0) \% & 60.75 & 67.6 & 66.4 & 77.2 & 70.8 \\
\hline Right medial forefoot (A1) \% & 60.5 & 65.2 & 67.8 & 71.5 & 69.8 \\
\hline Right lateral forefoot (A3) \% & 86.9 & 90.8 & 91.1 & 96.5 & 91.4 \\
\hline Right toe (A2) \% & 55.9 & 62.3 & 64.2 & 67.7 & 65.5 \\
\hline Left heel (A4) \% & 61.3 & 66.8 & 69.9 & 78.6 & 74.3 \\
\hline Right medial forefoot (A7) \% & 58.4 & 64.7 & 65.1 & 69.3 & 67.2 \\
\hline Left lateral forefoot (A5) \% & 86.5 & 92.3 & 94.6 & 98.4 & 93.7 \\
\hline Left toe (A6) \% & 58.8 & 65.5 & 64.8 & 70.1 & 67.6 \\
\hline BPPV: benign paroxysmal positional vertigo & & & & &
\end{tabular}

Table 3. Parameters found significant between the patient group with peripheral vestibular pathology and normal subjects to differentiate normal from pathology

\begin{tabular}{|c|c|c|c|}
\hline & Normal & Vestibular pathology & $p$ values \\
\hline Average step length (m) & $0.64 \pm 0.06$ & $0.56 \pm 0.09$ & $0.0000^{*}$ \\
\hline Step symmetry in the distance walked & $1.23 \pm 0.69$ & $2.23 \pm 1.65$ & $0.0000^{*}$ \\
\hline Back of the waist anterior-posterior (Deg) & $8.08 \pm 1.32$ & $10.40 \pm 3.94$ & $0.0000^{*}$ \\
\hline Tilt angle during gait (Deg) & $6.57 \pm 1.08$ & $2.83 \pm 1.03$ & $0.0000^{*}$ \\
\hline Max. left knee bending angle (Deg) & $63.57 \pm 9.38$ & $50.40 \pm 2.08$ & $0.0000^{*}$ \\
\hline Max. left lateral swing (Deg) & $13.80 \pm 2.08$ & $17.44 \pm 6.49$ & $0.0000^{*}$ \\
\hline Max. right knee bending angle (Deg) & $60.13 \pm 7.62$ & $49.83 \pm 12.76$ & $0.0000^{*}$ \\
\hline Max. right lateral swing (Deg) & $13.61 \pm 2.60$ & $18.58 \pm 6.56$ & $0.0000^{*}$ \\
\hline
\end{tabular}

Table 4. Correlation analysis of walking parameters between normal subjects and individual vestibular pathologies

\begin{tabular}{|c|c|c|c|c|}
\hline Normal subjects & $\begin{array}{l}\text { BPPV } \\
\text { ( } p \text { and } r \text { values) }\end{array}$ & $\begin{array}{l}\text { Meniere's disease } \\
\text { ( } p \text { and } r \text { values) }\end{array}$ & $\begin{array}{l}\text { Vestibular neuritis } \\
\text { ( } p \text { and } r \text { values) }\end{array}$ & $\begin{array}{l}\text { Unilateral vestibular hypofunction } \\
\text { ( } p \text { and } r \text { values) }\end{array}$ \\
\hline Walking speed $(\mathrm{m} / \mathrm{s})$ & $0.003 / 0.597$ & 0.020 & $0.036 / 0.664$ & $0.001 / 0.779$ \\
\hline Average step length (m) & $0.047 / 0.782$ & $0.037 / 0.669$ & $0.041 / 0.652$ & $0.001 / 0.780$ \\
\hline Step symmetry in the distance walked & $0.041 / 0.419$ & $0.001 / 0.512$ & $0.001 / 0.469$ & $0.001 / 0.686$ \\
\hline Step speed $(\mathrm{m} / \mathrm{s})$ & 0/0.791 & - & $0.041 / 0.652$ & $0.001 / 0.750$ \\
\hline Back of the waist anterior swing (Deg) & $0.04 / 0.431$ & $0.043 /-0.592$ & - & - \\
\hline Back of the waist posterior swing (Deg) & - & - & - & - \\
\hline Tilt angle during gait (Deg) & $0.033 / 0.446$ & - & - & - \\
\hline Back of the waist lateral swing right (Deg) & - & $0.01 / 0.708$ & - & - \\
\hline Max. right knee bending angle (Deg) & $0.005 / 0.887$ & $0.034 / 0.614$ & $0.041 /-0.651$ & - \\
\hline Max. right lateral swing (Deg) & $0.006 / 0.467$ & - & - & $0.023 /-0.583$ \\
\hline
\end{tabular}


a significant correlation was detected in walking speed, average step length, step symmetry in the distance walked, step speed, back of the waist lateral swing max., max. left lateral swing, max. right lateral swing (Table 4). Apart from walking speed and step speed, none of the features displayed significant difference. For the differentiation of the pathologic side in different disorders, the most significant features were left and right step symmetry in the distance walked, left and right back of the waist lateral swing. The dissociation rate of normal and pathological subjects was found to be $99.4 \%$. The rate of separation of pathologies within themselves was $94.4 \%$.

\section{DISCUSSION}

Walking is the most important factor in human dynamic balance. Several spatial position and mobilisation parameters exist in gait data, such as anterior-posterior and lateral swing angle, gait speed and symmetry (9). Extracting and processing data related to gait provide critical clues to understand dynamic balance and reveal underlying pathologies (10). In this study, we evaluated the features of balance and walking. We detected that anteriorposterior body swing angle values of male and female subjects were similar to those in the literature (11). Also, the results of our study were aligned with those in the literature regarding lateral swing limit values for both genders. Jarchi et al. (12) recorded body mobility with accelerometers and extracted walking parameters. In our study, the significant correlation between body swing limits and ankle mobility was consistent with the findings of Jarchi et al. (12). In our patients, we detected a significant difference in left/ right step symmetry in the distance walked and maximum waist lateral sway according to the pathologic side. In patients with left/right vestibular pathology, the left/right sway was prominent. When the walking characteristics of the patients with different vestibular pathology were evaluated individually, in the BPPV and MD groups waist lateral sway right/left measurements, again in MD average step length and step speed, and in the VN group step symmetry in the distance walked, and step speed measurements were significantly different from normal subjects. However, in the patients with UVH, none of the walking parameters was significantly different (Table 3).

Ankle mobility is important for choosing the falling strategy and vestibular data to be used in the regulation of walking (10). In our study, this data was processed with motion sensors placed on the toe and, the usage level of the ankle in the walking cycle was established. The significant correlation found between ankle mobility and the knee bending angle in our study will contribute greatly to identifying walking problems that can emerge, especially with lower extremity and balance problems (Table 4). In previous studies, only the effect of the ankle was determined; the knee joint was not considered (13-15).

Examination of the step lengths and widths of the subjects produced results similar to previous studies. The stepping width and step speed were established as important clues for walking and some pathologies (16). Similarly, our study found a significant relationship between step width-stepping speed and body anterior swing angle, body lateral swing limit angles and ankle mobility. Angunsri et al. (17) studied subjects with various vestibular pathologies and exhibited differences regarding step speed, symmetry and width. Data from our study revealed that the relations between walking pattern and body stability are consistent with the previous studies. Our study revealed that the average step length in BPPV, MD, VN, UVH and normal subjects were significantly different (Table 4). The BPPV group's average step length was lower and knee bending angle was higher than those of the MD, VN and UVH groups. It was emphasised that Borel in MD, Kubo in VN, Horak in patients with unilateral vestibular involvement had a significant deterioration in walking parameters such as step length and step speed, especially in the acute period (18-20). Demian found that patients with acute vestibular disorders generally kept balance by sustaining their step length short and extending the arms (21). In our study, similar findings were obtained, and it was seen that patients in the acute period felt safer by keeping their step length short, similar to the acute phase patients in Demian et al. (19) study. Also, the relationship between the parameters of the groups with peripheral vestibular pathology was examined, which demonstrated that in patients with BPPV, the increase in the average step length resulted in the distortion of the step symmetry, increase in the knee bending angle and waist anterior sway. Similar findings were also found in UVH group patients (Table 4).

Data from ankle sensors allows predicting symmetrical walking pattern, the motion of feet with relation to earth's axis, feet symmetry and height and the relation between toes up and falling and balance loss (11). Our equipment collected data simultaneously, such as duration, pattern, lifting off the floor, the symmetry between feet, angular direction, whereas computerised dynamic posturography cannot generate. In unilateral vestibular pathologies, locomotor activity deviates toward the pathology direction (17). The level of impairment in walking speed and symmetry (foot sensors 1\&2), the percentage increase in the use of the foot sensor on the side of the pathology gives information on the pathology's degree (Table 2). Ankle sensors detect differences in the distance between feet, regularity, deviation towards a certain direction and obtain information about the degree and side of the pathology (Table 4).

In peripheral vestibular lesions, knee dorsiflexion is impaired, lateral vestibulo-spinal and relevant tracts are affected (22). Data from knee sensors, such as maximum movement angle and lateral swing degree of the knee, enables the detection of problems in motor tracts. With anterior-posterior angle data obtained from knee sensors (sensors 3-4), the knee bending angle of the individual during dynamic walking was calculated. Calculation of the lateral movement angle of the knee can help detect the impact of problems arising from the knee joint on walking and dynamic balance (Table 4). 
The sensor on the back of the waist (sensor 5) provides information on the change of the centre of gravity (COG) and leaving the centre during active walking. The impaired ability to walk in a straight line, often seen in unilateral vestibular weaknesses, can easily be observed. This data helps diagnose unilateral and bilateral vestibular weakness. The anteriorposterior and lateral swing degrees obtained from the sensor on the back of the waist enable access to COG. The $z$ - and $y$-axis information provides COG data and allows for comment on the sensory organisation (23). Body lateral sway angles provide detailed and instant information on rhythmic load transfer strategy, which is one of the most challenging activities for individuals with vestibular problems. The data obtained in our study demonstrated that the amount of anterior-posterior sway was higher in the pathological group; however, waist lateral sway values were lower. O'Sullivan et al. (24) found that anteriorposterior sway was higher in patients with vestibular pathology, which was higher than lateral sway. In addition, if the patient's anteroposterior stability limit was reduced, walking with short steps and if the mediolateral stability limit was decreased, a broad-based walking pattern was detected. Our study was in accordance with the current literature and provided information about the body sway amount and limits in the normal and pathological group (Table 4).

Sensors in the insole recorded data related to changes in the plantar load distribution during gait, plantar load transfer between feet, symmetric distribution of load, calculation of load per foot during gait, forming even pressure area on plantar surface and distribution during gait, feet involvement in dynamic balance and can be compared with gyroscope data from ankle sensors to establish gait symmetry, walking speed, moving away from the centre and plantar pressure. Crea analysed walking patterns of healthy individuals with plantar sensors to determine the body stability and distribution of plantar pressure. The data collected from plantar sensors in our study support this, revealing a correlation between toe sensors and lateral swing angle values (25). Also, we showed a high correlation between data obtained from toe sensors and step length data obtained from motion sensors. Moreover, we demonstrated a correlation between rhythmic weight transfer symmetry and the load on foot. Motion sensor data on gait asymmetry towards a direction and plantar usage percentage differences were also highly correlated. Foot sensors can also provide information on walking stability, pressure differentiation, impairment in pressure distribution on static standing, impaired continuity in dynamic walking, plantar pressure distribution and pressure centre. Maximum usage values for dynamic walking were examined for each sensor, and the general gait line and the loaded line were determined. The most crucial gait parameters in the literature are distance, speed, gait pattern and independence (26). In our study, data related to these and additional parameters were successfully determined. Walking, regularity and speed data were established on a subject basis by examining the duration of the pressure applied on each sensor. Therefore, walking symmetry was calculated for both feet via pressure sensors (Tables 2, 4).

The comparison of subjects with/without pathologies can yield detailed information on the location and characteristic of the pathology. Several studies that obtained data on decrease in step speed, the decline in the general speed of walking, narrowangle at the ankle on heel's contact with the floor, changes in ankle angle and heel pressure with increasing pathology severity provided findings supportive of our study $(9,17,22,27-29)$. In our study, patterns related to walking speed and the body's sway limits were examined, in subgroups with peripheral pathology, walking pattern and body stability parameters were significantly different. In the literature, examination of the variables in the posture and swing phase among normal subjects and patients with peripheral vestibular pathology revealed that walking pattern played an effective role in diagnosing vestibular pathology (Table 4) $(16,17)$.

The success of the system in the binary (healthy-pathologic) classification was $99.4 \%$, and the success in the disease classification was $94.4 \%$. Thus, the total performance is evaluated as $93.8 \%$. On the other hand, it was observed that there were three self-attributes among the correlations of force, motion and force-motion sensor data, which contribute to our predictions about the original aspect of the project (Table 3). According to our study results, our algorithm has three main determinants of step length/speed, step symmetry/pressure distribution and angular values of body swing in recognising/classifying vestibular pathologies.

\section{Study Limitations}

Normalisation data in our study will provide critical clues for distinguishing between individuals with and without disorders. Dynamic balance and walking analysis by our system are considered important in audiology, orthopaedics and neurology. It can contribute clinically by collecting data from several patients with different pathologies. The most important limitations of our study were the absence of normal individuals in different age groups, normalisation and classification in large subject groups with different pathologies (bilateral vestibular weakness, neurological diseases, stroke, orthopaedic disabilities, cerebral palsy, different gait disorders, others).

\section{CONCLUSION}

In this study, normal and pathological conditions could easily be distinguished by our algorithm (healthy-pathological classification reliability: 99.4\%). In the pathological group, although working in a small sample size various disorders have been differentiated with $94.4 \%$ accuracy. Our system could reliably measure even minor differences that could not be detected by other conventional equipment.

Ethics Committee Approval: The study has been approved by the İstanbul University Cerrahpaşa Faculty of Medicine with the approval number A-57 (date: 07.07.2015). 
Informed Consent: Written consent was obtained from the participants before the study.

Peer-review: Externally peer-reviewed.

Author Contributions: Concept - E.K., S.İ., A.A.; Design - E.K., S.İ., A.A.; Data Collection and/or Processing - E.K., K.Ş., T.Ç.; Analysis and/or Interpretation - E.K., S.I., K.Ş., T.Ç., A.A.; Literature Search - E.K., K.Ş., T.Ç.; Writing - E.K., S.I., A.A.

Conflict of Interest: The authors have no conflict of interest to declare.

Financial Disclosure: This study was supported as part of TUBITAK 1001 project no: 115E258, patent number: TR $201317711 \mathrm{~B}$.

\section{REFERENCES}

1. Ferrè ER, Bottini G, lannetti GD, Haggard P. The balance of feelings: vestibular modulation of bodily sensations. Cortex 2013; 49: 748-58.

2. Palm HG, Lang P, Strobel J, Riesner HJ, Friemert B. Computerized dynamic posturography. Am J Phys Med Rehabil [Internet] 2014; 93: 4955.

3. Nesti A, Barnett-Cowan M, Macneilage PR, Bülthoff HH. Human sensitivity to vertical self-motion. Exp Brain Res 2014; 232: 303-14.

4. Şimşek D, Ertan H. Control and sport: muscular fatıgue and postural control relationship. Spormetre Beden Eğitimi ve Spor Bilim Derg 2011; 9: 119-24.

5. Bertolini G, Straumann D. Moving in a Moving World: A Review on Vestibular Motion Sickness. Front Neurol 2016; 15; 7:14.

6. Mast FW, Preuss N, Hartmann M, Grabherr L. Spatial cognition, body representation and affective processes: the role of vestibular information beyond ocular reflexes and control of posture. Front Integr Neurosci 2014; 8: 44. doi: 10.3389/fneur.2016.00014.

7. Hegde N, Bries M, Sazonov E. A Comparative Review of FootwearBased Wearable Systems. Electronics. 2016; 5. https://doi.org/10.3390/ electronics5030048 [E-pub online ahead]

8. Roetenberg D, Luinge H, Slycke P. Xsens MVN : Full 6DOF Human Motion Tracking Using Miniature Inertial Sensors. Technical report. 2013. Available from: http://en.souvr.com/product/pdf/MVN_white_paper.pdf

9. Dye DC, Eakman AM, Bolton KM. Assessing the validity of the dynamic gait index in a balance disorders clinic: an application of Rasch analysis. Phys Ther 2013; 93: 809-18.

10. Bent $L R$, Inglis JT, McFadyen BJ. When is vestibular information important during walking? J Neurophysiol 2004; 92: 1269-75.

11. Shull PB, Jirattigalachote W, Hunt MA, Cutkosky MR, Delp SL. Quantified self and human movement: a review on the clinical impact of wearable sensing and feedback for gait analysis and intervention. Gait Posture 2014; 40: 11-9.

12. Jarchi D, Wong C, Kwasnicki RM, Heller B, Tew GA, Yang GZ. Gait parameter estimation from a miniaturized ear-worn sensor using singular spectrum analysis and longest common subsequence. IEEE Trans Biomed Eng 2014; 61: 1261-73.

13. Howell AM, Kobayashi T, Hayes HA, Foreman KB, Bamberg SJ. Kinetic gait analysis using a low-cost Insole. IEEE Trans Biomed Eng 2013; 60: 3284-90.

14. Mariani B, Rouhani H, Crevoisier X, Aminian K. Quantitative estimation of foot-flat and stance phase of gait using foot-worn inertial sensors. Gait Posture 2013; 37: 229-34.

15. Riaz $\mathrm{Q}$, Tao G, Krüger B, Weber A. Motion reconstruction using very few accelerometers and ground contacts. Graphical Models 2015; 79: 23-38.

16. Khandelwal S, Wickstrom N. Gait event detection in real-world environment for long-term applications: Incorporating domain knowledge into time-frequency analysis. IEEE Trans Neural Syst Rehabil Eng 2016; 24: 1363-72.

17. Angunsri N, Ishikawa K, Yin M, Omi E, Shibata Y, Saito T, et al. Gait instability caused by vestibular disorders - analysis by tactile sensor. Auris Nasus Larynx 2011; 38: 462-8.

18. Borel L, Harlay F, Lopez C, Magnan J, Chays A, Lacour M. Walking performance of vestibular-defective patients before and after unilateral vestibular neurotomy. Behav Brain Res 2004; 150: 191-200.

19. Demain A, Westby GW, Fernandez-Vidal S, Karachi C, Bonneville F, Do $\mathrm{MC}$, et al. High-level gait and balance disorders in the elderly: a midbrain disease? J Neurol 2014; 261: 196-206.

20. Kubo T, Kumakura H, Hirokawa Y, Yamamoto K, Imai T, Hirasaki E. 3D analysis of human locomotion before and after caloric stimulation. Acta Otolaryngol. 1997; 117: 143-8.

21. Horak FB, Dozza M, Peterka R, Chiari L, Wall C 3rd. Vibrotactile biofeedback improves tandem gait in patients with unilateral vestibular loss. Ann N Y Acad Sci 2009; 1164: 279-81.

22. Barker SP. Changes in gait, balance, and function with vestibular rehabilitation [Internet]. Vol. 3126514, ProQuest Dissertations and Theses. 2004. Available from: http://search.proquest.com/do cview/305179306?accountid=14701\%5Cnhttp://sfx.scholarsportal.info/ ottawa?url_ver=Z39.88-2004\&rft_val_fmt=info:ofi/fmt:kev:mtx:diss ertation\&genre $=$ dissertations $+\% 26+$ theses \&sid=ProQ:ProQuest $+D$ issertat ions+\%26+Theses+Global\& atitle

23. Alberts JL, Hirsch JR, Koop MM, Schindler DD, Kana DE, Linder SM, et al. Using Accelerometer and Gyroscopic Measures to Quantify Postural Stability. J Athl Train 2015; 50: 578-88.

24. O'Sullivan M, Blake C, Cunningham C, Boyle G, Finucane C. Correlation of accelerometry with clinical balance tests in older fallers and nonfallers. Age Ageing 2009; 38: 308-13.

25. Crea S, Donati M, De Rossi SM, Oddo CM, Vitiello N. A wireless flexible sensorized insole for gait analysis. Sensors (Basel) 2014; 14: 1073-93.

26. Bohannon RW, Horton MG, Wikholm JB. Importance of four variables of walking to patients with stroke. Int J Rehabil Res 1991; 14: 246-50.

27. Lang J, Ishikawa K, Hatakeyama K, Wong WH, Yin M, Saito T, et al. 3D body segment oscillation and gait analysis for vestibular disorders. Auris Nasus Larynx 2013; 40: 18-24.

28. Liu T, Inoue Y, Shibata K. Development of a wearable sensor system for quantitative gait analysis. Meas J Int Meas Confed 2009; 42: 978-88.

29. Hung TN, Suh YS. Inertial sensor-based two feet motion tracking for gait analysis. Sensors (Basel) 2013; 13: 5614-29. 